\title{
Femmes de Lia (Épire) entre 1935 et 1948
}

D’après le livre de Nicholas Gage « Eleni »

Women of Lia (Epirus) from 1935 to 1948

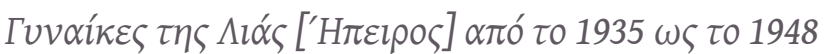

Joëlle Dalègre

\section{OpenEdition \\ Journals}

Édition électronique

URL : https://journals.openedition.org/ceb/3974

DOI : $10.4000 / c e b .3974$

ISSN : 2261-4184

\section{Éditeur}

INALCO

Édition imprimée

Pagination : 229-237

ISBN : 978-2-85831-205-4

ISSN : 0290-7402

Référence électronique

Joëlle Dalègre, «Femmes de Lia (Épire) entre 1935 et 1948 », Cahiers balkaniques [En ligne], 41 | 2013, mis en ligne le 19 mai 2013, consulté le 06 juillet 2021. URL : http://journals.openedition.org/ceb/3974 ; DOI : https://doi.org/10.4000/ceb.3974

Ce document a été généré automatiquement le 6 juillet 2021.

\section{(c) (1) (8)}

Cahiers balkaniques est mis à disposition selon les termes de la Licence Creative Commons Attribution - Pas d'Utilisation Commerciale 4.0 International. 


\title{
Femmes de Lia (Épire) entre 1935 et 1948
}

\author{
D’après le livre de Nicholas Gage « Eleni » \\ Women of Lia (Epirus) from 1935 to 1948

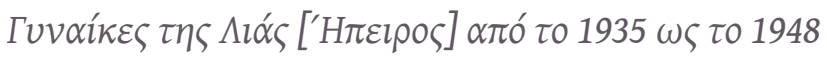

Joëlle Dalègre

1 Ce livre entre dans la catégorie des « récits de vie », récit de seconde génération, écrit par un fils qui cherche dans son enquête des explications à l'exécution de sa mère 30 ans plus tôt. Le résultat de cette quête a été un livre provoquant parce qu'écrit par un témoin trop jeune au moment des faits pour avoir tout compris et mémorisé, par un témoin partial, car devenu un anticommuniste notoire, et parce que ce livre a été réduit en un film du même titre d'un anticommunisme primaire et exploité par la droite tant américaine que grecque. Mais c'est un livre qui parle de femmes qui ne sont ni hérö̈nes, ni politiques, ni même féministes, aux prises avec la tourmente de la guerre, de l'Occupation et de la guerre civile. Je vais donc présenter les spécificités de ce texte et du lieu avant d'étudier les femmes elles-mêmes.

\section{L'auteur}

2 Nicholas Gatzoyannis, devenu Nicholas Gage aux États-Unis, naquit en 1939 à Lia en Épire, $5^{\mathrm{e}}$ enfant et $1^{\mathrm{er}}$ fils d'Eleni, mariée en 1930 à Christos, qui travaille en Amérique et fait des enfants à sa femme à chaque retour au pays. En août 1948, sa mère qu'il adore est torturée puis mise à mort par des membres de l'Armée démocratique, car elle a refusé de laisser partir ses enfants dans les pays de l'Est et en a fait passer quatre, clandestinement, en zone gouvernementale, et se trouve donc « traître ». En mars 1949, le père récupère ses enfants et les emmène aux États-Unis, à Worcester dans le Massachusetts. Nicholas reçoit une éducation américaine, en 1964, il obtient un diplôme de journalisme à Columbia, en 1970, puis il travaille dix ans comme journaliste d'investigation pour l'Associated Press, le Boston Herald Traveller, le Wall Street Journal et le 
New York Times. Il se fait alors connaître par deux best-sellers sur la mafia, The Mafia Is Not An Equal Opportunity, Employer and Mafia, U.S.A. Il contribue aussi à dénoncer la corruption du vice-président Spyro Agnew (qui doit démissionner) et, pendant le scandale du Watergate, il est le premier reporter à parler des enregistrements. Son expérience de journaliste lui sert pour une émission de TV et comme coscénariste, puis producteur, du Parrain III.

Il revient en Épire en 1963 et en 1969, et comprend finalement qu'il lui faut, pour ses propres besoins psychologiques, éclaircir les circonstances de la mort de sa mère. En 1979, il demande donc à être envoyé comme correspondant à Athènes, puis prend un congé et, de 1980 à 1983, cherche à travers la Grèce et l'Europe communiste, les survivants de l'époque pour recueillir des témoignages ; il profite du retour en Grèce de certains exilés en 1982 pour en obtenir davantage. Il veut d'abord chercher les coupables de la mort de sa mère et la venger, puis lorsqu'il renonce à exécuter le coupable, dresser un monument à sa mère :

Le livre Eleni est un monument intellectuel pour le sacrifice de ma mère. Il traversera le temps et personne ne peut le détruire comme les monuments aux morts dans les villages grecs que les maires détruisent tous les 50 ans. Depuis que je l'ai perdue, à l'âge de 9 ans, j'ai eu conscience que je devais lui rendre les honneurs que je lui devais. Je suis devenu journaliste d'investigation pour développer mon habileté à localiser après tant d'années le juge qui a ordonné son exécution. Quand finalement je l'ai rencontré, j'ai trouvé devant moi un petit vieux, une ruine invalide. Il n'a rien reconnu, mais je suis parti en paix.

4 Le livre est publié en 1984. Traduit en 26 langues (32 selon une autre source), il reçoit le prix du meilleur livre de 1984 donné par la société littéraire royale de GB. Il donne naissance à un film en 1985, Eleni, avec John Malkowitz. Devenu dès lors un supposé « spécialiste » de la Grèce, Gage publie également,

A Place for Us ou les efforts de sa famille pour s'intégrer aux États-unis

The Bourlotas fortune

Hellas : A Portrait of Greece (un récit sur la Grèce des colonels)

Greece: Land of Light

Greek Fire: The Story of Maria Callas and Aristotle Onassis

Pourquoi ce livre a-t-il eu tant d'admirateurs et de détracteurs?

6 Il est le produit d'un anticommunisme virulent, écrit par un homme qui a été un petit garçon adorant sa mère qui a été exécutée par des communistes, et qui est élevé ensuite dans les États-Unis de Mac Carthy dont il adopte tous les codes pour être mieux intégré.

Il a été utilisé par tous les anticommunistes en Grèce et aux États-Unis.

8 En 1987 Reagan le cite dans ses discussions avec l'URSS à propos de la course aux armements.

9 Il arrive juste au moment où les anciens résistants, vaincus de la guerre civile, sortent $\mathrm{du}$ ghetto et $\mathrm{du}$ silence auxquels ils étaient condamnés, imposent leur vue des événements et ne veulent voir personne souiller cette image.

10 Même pour ceux qui reconnaissent que cette femme a été assassinée, il remet en avant la question du paidomazoma, du ramassage des enfants, qui reste un point chaud de l'historiographie grecque. 
11 Enfin N. Gage est resté fidèle à son anticommunisme virulent, s'est lié au parti de droite et aux nationalistes, a joué un rôle actif en Grèce comme conseiller politique de C. Mitsotakis (1990-94) et plus discrètement de Dora Bakoyanni et de A. Samaras, parce que devenu "spécialiste » des affaires épirotes, président honoraire de l'Association mondiale épirote, il est intervenu dans les différends gréco-albanais des années 1990 en faveur de la minorité grecque d'Albanie, en demandant une autonomie de l'Épire du nord; de plus, ses positions à propos des colonels sont fort discutées : il se félicite d'avoir publié dans le New York Times plusieurs articles contre les colonels, mais dans son livre sur la Grèce, il déclare que ce sont, à tout prendre, des dictateurs beaucoup moins dangereux que les communistes, qui vont remettre la Grèce dans le droit chemin.

\section{Lia en Thesprotie}

Le village de Lia est situé au NO de Ioannina ( $65 \mathrm{~km}$, autant d'Igoumenitsa), au pied du massif de la Mourgana ou Tsamada (du nom d'un village), près de la ligne qui sépare Grèce et Albanie (sommet: $1806 \mathrm{~m}$ ). Cette situation sur un passage à l'intérieur de la montagne et vers l'Albanie explique que soient passés par ce petit village, des voyageurs comme Lord Byron en 1810, ou plus récemment, Elia Kazan. K. Mitsotakis s'y est rendu le 22 juillet 1990 dans un contexte ardemment politique !

Cette position est stratégique entre 1941 et 1944 parce que le massif de la Mourgana pouvait bloquer l'acheminement des renforts allemands vers la côte épirote face à Corfou, qu'ils viennent d'Albanie ou d'Ioannina, et bloquer l'un des deux itinéraires d'évacuation que les Allemands pouvaient emprunter pour quitter la Grèce, supposé moins dangereux, via l'Albanie, que celui qui traversait toute la Yougoslavie. Pendant la guerre civile, il permet l'accès à l'Albanie proche, et, refuge de l'Armée démocratique, il est le dernier massif tenu par les andartès avec le Grammos. Enfin, autre particularité, N. Gage affirme que c'est la proximité du camp de Kalpaki, où Métaxas envoyait discipliner les recrues soupçonnées de communisme, qui aurait initié au communisme les premiers jeunes du village.

14 Les habitants de Lia sont donc sans cesse confrontés à la guerre et à des enjeux qui les dépassent. En octobre 1941, une petite patrouille italienne occupe le village, mais, à peine un mois plus tard, les villageois voient de loin sa défaite et son départ. Après la victoire allemande, les Italiens reviennent et, chargés de la zone, se contentent d'envoyer périodiquement au village des patrouilles chercher de possibles caches d'armes, ils placent enfin au poste de police quatre Grecs collaborationnistes. Les premiers résistants du village, en mars 1943, encerclent ce poste dont les gendarmes se rendent sans difficulté et, le lendemain, annoncent au village un ordre nouveau, celui de l'ELAS. Dès lors, le village devient une petite Moscou aux mains d'un petit groupe communiste qui se rend rapidement maître des avant-postes italiens dont les hommes se regroupent à Filiates. La Mourgana ne voit plus l'envahisseur. Mais, pendant l'été 1943, la région est au coeur des combats ELAS/EDES, l'EDES s'implante à proximité, mais ne monte pas dans la montagne, où Lia, Babouri, Tsamanda restent aux mains de l'ELAS. En juillet de la même année, les Britanniques installent à Lia la mission Bovington, 3 hommes pour surveiller l'ELAS et les relations entre ELAS et EDES. En octobre 1943, avec la fin de la courte trêve entre les deux groupes résistants, l'EDES victorieux entre dans Lia, mais le 14 novembre, l'ELAS réoccupe le village où, à 
Noël 1943, elle conduit le général italien Infante, pour que les Anglais le fassent passer en Albanie, puis en Italie. Entre le 2 et le 9 janvier 1944, le village change plusieurs fois de mains entre EDES et ELAS, avant de rester définitivement à l'ELAS. Le 26 février 1944, la mission britannique part. Un peu avant Pâques 1944, le commandant allemand de l'Épire, Hubert Lanz, prépare son offensive contre les andartès de la Mourgana pour dégager l'itinéraire. À la mi-avril, ses troupes arrivent à Lia, brûlent une partie du village et s'en vont, l'ELAS revient. Au printemps 1945, les premiers représentants du gouvernement grec reviennent à Lia et les anciens proches de l'ELAS prennent la montagne et passent en Albanie. Difficile de savoir où se trouve le pouvoir...

Le 27 novembre 1947, les 6 gendarmes en poste reçoivent l'ordre de partir face à l'arrivée de l'Armée Démocratique, qui s'installe à Lia jusqu'au 18 septembre 1948. Pendant ces 10 mois, le village est sans arrêt aux avant-postes de combats qui se déroulent parfois à quelques kilomètres, passe l'hiver 1948 sous le bruit quotidien des spitfire et des bombardiers. En février 1948, c'est l'opération Pergame, les gouvernementaux s'arrêtent aux portes de Lia, une nouvelle fois en avril (opération Faucon), puis une nouvelle opération, Taureau, lancée en septembre 1948 permet aux gouvernementaux de reprendre la région. Eleni est exécutée le 28 août, le 12 septembre, l'Armée démocratique évacue obligatoirement toute la population vers l'Albanie (ne resteront à Lia qu'une vieille aveugle et une femme cachée dans un ravin), le 18 septembre les troupes gouvernementales entrent dans le village.

\section{Les femmes de Lia}

\section{Avant la guerre}

C'est une société fermée, où on a peu de contacts avec l'extérieur, où la vie des femmes en particulier est régie par des règles strictes qu'elles paraissent accepter. Une description assez proche de celle que l'on trouve dans nombreuses études ethnographiques ${ }^{1}$.

Société majoritairement féminine, car une partie des hommes est absente, en Amérique, ou travaille comme rétameurs et tonneliers itinérants qui partent pour trois ans. Cependant leur présence est toujours sensible, car les femmes appliquent leurs instructions et gèrent avec la perspective de rendre des comptes à leur retour.

Société "fermée »: on voit que même Ioannina est inaccessible à la plupart des habitants qui ne se déplacent qu'à pied ou à mulet, que pour consulter un médecin de qualité, il faut aller à Corfou, soit $12 \mathrm{~h}$ de mulet avant d'atteindre la côte et d'ajouter la traversée. Les premiers trajets en camion en 1945 et 1948 terrorisent les enfants de la famille. En allant à Corfou, Eleni découvre une architecture, une végétation inconnues, la mer, mais aussi une paire de chaussures et une robe de style européen, c'est là que son mari achète le lit qui fera scandale au village. On voit aussi les villageois stupéfaits devant les habitudes anglaises, les shorts, la viande en boite, les nourritures en poudre, le cacao, la radio...

19 Les femmes exécutent la totalité des tâches domestiques à l'intérieur de la maison et de sa cour, et un petit nombre seulement à l'extérieur pour limiter les contacts avec les hommes, et seulement si elles sont fillettes prépubères ou femmes mariées. La jeune 
Olga est ravie de la messe de la Chandeleur, première occasion depuis celle de Noël de sortir de la maison, parce qu'elle a 15 ans !

«Que vos enfants soient des mâles et vos chevreaux des femelles!» se souhaitaient les villageois en levant leur verre de tsipouro. Un enfant de sexe féminin était un boulet à traîner, car elle contraignait ses parents à veiller sur sa vertu et à constituer une dot jusqu'à ce qu'elle parte, jeune épousée vivre sa vie ailleurs en travaillant pour la famille du mari ». (p.50-51) L'ensemble des règles a pour but de conduire une fille au mariage une fille vierge qui ne nuira pas à l'honneur de la famille: «l'honneur n'a ni prix ni mesure. Une femme n'a pas de plus grand trésor. » (p.63)

Une fillette coiffait le fichu dès l'âge de onze ans, afin que nulle boucle rebelle n'éveille le désir d'un inconnu. Elle n'avait pas le droit de s'adresser à un homme ne faisant pas partie de sa famille, même pas pour un simple bonjour. La jeune fille n'apparaissait en public que deux fois l'an, à l'occasion de l'office de Noël et de Pâques où elle demeurait au milieu des femmes. Le reste du temps, à l'abri des murs de pierre et sous l'œil vigilant de son père et de ses frères, elle s'initiait aux tâches féminines : soigner les animaux et s'occuper des champs, couper le bois, faire la cuisine et le ménage, coudre, filer et broder son trousseau. La beauté et la vertu ne pesaient que de peu de poids au regard d'une dot trop modeste... Quand un mariage était conclu par les intermédiaires, la jeune mariée était portée jusqu'au domicile d'un homme à qui elle n'avait jamais adressé la parole, et dont parfois le visage même lui était inconnu, et elle passait sa nuit de noces, non pas aux côtés de son époux, mais de sa belle-mère pour symboliser sa sujétion. Son initiation sexuelle, parfois accomplie de force dans l'étable, pouvait être terrifiante, avec 4 générations vivant dans une maison de 2 pièces, l'intimité était chose rare. (p.50-51)

Une fois mariée, il lui restait à honorer la famille de son époux, par son travail, son obéissance, ses enfants mâles (p.79 "elle avait enfin atteint l'objectif de sa vie de femme » dit-on d'Eleni quand elle a enfin un fils) et sa vertu. Eleni, une très belle jeune fille, fille d'un meunier, épouse à 19 ans, Christos, le $5^{\mathrm{e}}$ fils d'une famille, 14 ans de plus qu'elle, revenu pour ce faire des États-Unis; elle vit chez et avec sa belle-mère jusqu'à la mort de cette dernière en 1935. Personne ne lui a demandé son avis pour le mariage et lorsque l'accord s'est fait entre le mari et le père ( $\mathrm{p} 44)$ «son père lui ordonna d'apporter le café traditionnel. Elle ne regarda même pas le visage de l'étranger quand il prit sa tasse et pose à sa place un billet de 20 dollars américains en disant : c'est pour toi. Elle savait qu'on avait décidé de son avenir ». Mais, Eleni a de la chance dit-on, car son mari ne la bat pas, il envoie régulièrement de l'argent des États-Unis et revient, nette bénédiction par rapport à celles dont le mari est mort, ou prend une nouvelle femme ailleurs et qui attendent jusqu'à leur mort un retour... De surcroît ce mari gagne bien sa vie et permet des achats extraordinaires : une machine à coudre Singer et un gramophone, un pot en argent de Constantinople, ou, encore plus, un lit... qui pour elle est une nouveauté inutile au point qu'elle continue à coucher par terre et ne l'utilise que quand le mari est là. Le lit est d'ailleurs considéré par les autres, au village, comme un superflu insupportable. Le mari a doublé la taille de la maison, 4 pièces au lieu de 2, ce qui lui vaudra pas mal de jaloux, il a installé une entrée distincte pour les animaux et une douche extérieure avec des tonneaux. Cette belle maison unique sera, pour cela même, réquisitionnée par les dirigeants locaux de l'Armée démocratique, puis transformée en tribunal et lieu de torture et de détention en 1948. Mais on dit d'Eleni qu'elle est «taxidemeni », « amérikana », le premier signifie que son mari est au loin et 
qu'il faut donc surveiller de plus près encore son comportement, le second qu'on la jalouse malgré ses efforts pour ne pas donner prise à l'envie...

Pourquoi ne pas aller en Amérique ? Eleni demande d'abord à rester pour prendre soin de ses vieux parents et de sa sœur. Mais quand elle demande à plusieurs reprises, à rejoindre son mari, il lui répond (p.59) : «toutes des putains en Amérique ! Ce n'est pas un endroit pour les femmes honnêtes!», «ce n'est pas un endroit pour les enfants, en particulier si ce sont des filles » (p.62), car :

Quand il fut marié et eut des enfants, il décida que les États-Unis étaient un pays beaucoup trop périlleux pour y élever une famille, en particulier quatre filles. Travaillant de longues heures tous les jours, il n'aurait pu les surveiller correctement et sa femme aurait souffert de l'absence du réseau de proches et de voisines qu'elle avait à Lia. Au village, épouses et filles savaient exactement comment se conduire; les mœurs rigides ne toléraient pas le moindre écart, alors que l'Amérique fourmillait de femmes déchues. (p.61)

\section{Face à la guerre} habituelles ne fournissent pas de solutions. Tout le monde est touché, mais les femmes davantage, car les hommes peu à peu disparaissent du village, ceux qui étaient en tournée au loin ne rentrent pas, ceux qui se sont engagés dans l'EDES ou l'ELAS fuient pour éviter les représailles quand l'autre groupe s'approche comme ceux qui, en 1945, fuient les gouvernementaux, ceux qui ne veulent pas être enrôlés dans l'une ou l'autre armée pendant la guerre civile s'enfuient également.

peur est quasi permanente et l'on peut dire justifiée car la violence est présente à chaque page et dans tous les camps ; à deux reprises, l'arrivée des Italiens puis celle des Allemands, les familles en leur totalité fuient vers la montagne s'installer dans des cavernes proches de l'Albanie, le fait d'être proche des combats ELAS/EDES et des combats $\mathrm{AD} /$ Armée gouvernementale répand également une inquiétude permanente, les familles sont sommées de rejoindre les maisons du bas du village ou, au contraire, du haut, selon l'avancée de l'un ou l'autre groupe, avant l'évacuation finale forcée de septembre 1948. Comme les hommes restants sont souvent vieux ou malades, ce sont les femmes qui portent les biens, couvertures, provisions, même les enfants ou les personnes âgées qu'il faut emmener sur des brancards à chaque évacuation. Il n'y a pas que le port héroïque des munitions.

Les femmes doivent affronter, comme partout en Grèce, le manque de nourriture. Le village a quelques ressources, mais l'argent des travailleurs migrants permettait d'ajouter des réserves, morue séchée, olives, fruits secs, riz, café. Tout cela est introuvable, il faut donc recourir aux ressources de la montagne, herbes, escargots, champignons, tortue, impossible de chasser puisque les armes sont interdites. Les plats communs deviennent le kofto, du froment pilé en riz, l'infusion d'orge grillée, le pain grossier de farine dit bobota, la shilira : bouillie faite de lait de chèvre épaissie avec du yaourt et de la levure qui a reposé une journée. Les femmes doivent également effectuer les travaux agricoles que faisaient les hommes ou des employés. Celles qui n'ont plus de réserves ou des terres trop petites vont à 2 jours de marche ramasser du sel sur la côte pour le vendre en Albanie où d'autres, à pied toujours, vont troquer des objets précieux contre du blé ou du maïs, ou s'embauchent pour la récolte des olives. L'arrivée d'une mère et de ses filles venues d'Athènes, montre que la famine est encore 
plus forte en ville, même si la tuberculose et le froid sévissent, Olga souffre d'un goitre faute d'iode dans l'alimentation.

En 1945 la pression alimentaire diminue : chaque mois une cargaison de l'UNRRA est déchargée à Igoumenitsa, un comité transporte des rations à Lia pour ceux qui ne peuvent faire les $8 \mathrm{~h}$ de trajet de Lia à Filiates pour participer à la distribution, huit livres de farine et une demie de sucre/personne, $200 \mathrm{~g}$ de riz, autant de lentilles, 2 boites de lait concentré/enfant, lait en poudre, œufs, et parfois conserves, thé, biscuits ou chocolat. Mais l'année 1947-48 est à nouveau une année marquée par la faim en raison des réquisitions de l'Armée démocratique qui doit subvenir à ses besoins coûte que coûte. Le manque de vêtements et de chaussures devient également criant après 8 ans de guerre, au point que les sœurs de Nicolas marchent pieds nus régulièrement.

Les femmes découvrent l'inconnu, sans repère dans leurs acquis pour y faire face, elles jugent et décident souvent en s'accrochant à leurs valeurs sûres qui sont inadaptées à ce monde nouveau. Comment juger les jeunes filles venues de la ville?

Elles portaient une robe imprimée en tissu collant, visible sous leur léger manteau de drap et leurs chapeaux attiraient tous les regards. En dehors des grandes villes, aucun villageois n'avait jamais vu de femme en cheveux, mais ces deux-là avaient des boucles qui se balançaient impudiquement sur les épaules, des cheveux libres sous les yeux du Christ tout-puissant qui dardait sur elles un regard noir du haut du dôme de la voûte centrale. (p.86)

De plus elles saluent les hommes dans la rue, ne baissent pas les yeux, alors, quand elles acceptent, pour ne pas mourir de faim, de faire la cuisine et le ménage des trois Anglais de la mission, on les prend pour des putains. Eleni reste fidèle à ses codes, quand elle doit envoyer une de ses filles garder les troupeaux, elle choisit celle de 8 ans qui ne fera pas jaser à ses dépens, quand l'UNRRA en 1945 donne à Kanta une petite robe bleue avec des manches bouffantes courtes, elle l'oblige à la poser et dit «tu ne vas pas rentrer au village dans cet accoutrement! Que vont penser les gens en voyant une fille de treize ans montrer à tout le monde ses bras nus?» (p 167). Olga, en 1947, à 17 ans, porte le fichu serré comme une nonne et se couvre les joues de cendre pour ne pas attirer les regards des combattants cantonnés au village. Quand, en 1945, Eleni reçoit des nouvelles et de l'argent de Christos qui lui dit de marier son aînée avant de le rejoindre en Amérique, elle doit choisir entre payer le notaire pour les papiers (les registres de Lia ont été brûlés) ou acheter le trousseau de sa fille, elle obéit à son mari et achète le trousseau, donc elle reste...

31 Comment juger les événements? Personne n'est sûr de rien au village, on pense qu'en étant polis et accueillants avec les Allemands, on évitera le pire... on écoute les premiers andartès qui annoncent des réformes attirantes et on participe aux comités et aux réunions de formation ${ }^{2}$, on craint les soldats de l'EDES décrits comme abominables et puis... les Allemands brûlent le village et même l'église, l'ELAS exerce de terribles vengeances, les hommes de l'EDES se montrent fort civils, Christos lui-même, ignorant tout, écrit à sa femme qu'elle n'a rien à craindre de Grecs du village et doit donc rester chez elle. Les gens eux-mêmes changent, certains que l'on croyait sûrs, changent de camp, les amies calomnient, à qui se fier?

Le trouble s'accroît quand il s'agit de comprendre des femmes qui vivent un autre choix : les andartines! Ces jeunes filles mènent une vie inimaginable selon les critères traditionnels : elles portent un pantalon, des armes, passent la nuit dehors avec des hommes, elles jettent donc l'effroi quand on les aperçoit au village! Elles seront donc 
immariables à leur retour! Dans un premier temps, l'auteur nous les présente toujours comme des jeunes filles contraintes par la force, prêtes à tout pour y échapper (comme sa sœur qui se fait verser de l'eau bouillante sur le pied pour échapper au recrutement), ou contraintes par la nécessité absolue (femme sans homme qui ne veut pas mourir de faim), l'une d'elles explique que sa famille n'aura plus à la nourrir et obtiendra peutêtre un meilleur traitement. Quand, pour la première fois dans l'été 1947, Eleni et Olga voient, à Ioannina, des andartines du Pogoni, elles sont « en larmes, le visage écorché, la tête nue, les cheveux sales et emmêlés ", elles avaient été « enlevées de force » deux mois plus tôt, contraintes de porter un uniforme et de se battre et venaient de s'échapper pour rejoindre les gouvernementaux (p.199), leur pantalon kaki scandalise Olga bien évidemment. Quelque temps plus tard, une longue file d'andartines traverse Lia «des paysannes d'une vingtaine d'années, aux longues tresses épaisses... elles portaient un uniforme kaki et en particulier - les villageois avaient de la peine à en croire leurs yeux - un pantalon d'homme! Si elles avaient défilé nues, elles n'auraient pas causé une sensation plus forte! Nicolas, extrêmement perplexe, pensait que les soldats étaient mi-hommes, mi-femmes. » (p.225) Elles sont dans un état léthargique, un épuisement extrême, disent que dans leur village, on a enrôlé de force toutes les filles de plus de 15 ans. C'est là qu'olga et sa mère décident d'une blessure volontaire, Kanta, en décembre 1947, plus jeune et maigre, supposée ne pas attirer les regards masculins, répondra à l'appel pour sauver la famille. Quatre-vingts jeunes filles doivent s'entraîner au maniement des armes et aux marches forcées, dormir la nuit dehors dans le froid, manger peu, vivre dans la crasse, subir également un enseignement idéologique. Une semaine plus tard, elles reçoivent un uniforme, souvent taché de sang et la nuit du 26 décembre 1947, elles doivent partir d'urgence pour un village voisin et porter jusqu'à épuisement les mitrailleuses sur leur dos.

Force est cependant à Nicholas Gage de reconnaître que certaines engagées de force vont prendre goût à ce cadre nouveau, surtout dès qu'elles sont éloignées du village, l'une d'entre elles accepte de couper ses nattes, certaines semblent apprécier le maniement des armes, les séances de propagande, les discussions avec les guérillas. « À son grand étonnement, quelques filles semblaient ravies de leur nouvelle tenue et se pavanaient comme des hommes en levant leur fusil avec gaucherie dans un simulacre de virilité » (p.244). Vivre sur la Mourgana en 1948, c'est se trouver au cœur même des combats de la guerre civile et l'Armée démocratique, dans sa lutte désespérée, accentue donc sa pression sur les civils, réquisitions des vivres et des mulets, travail civil obligatoire, enrôlement forcé des jeunes des deux sexes et, enfin, le "paidomazoma ", l'évacuation des enfants vers l'Albanie. Pas d'hésitation ici dans l'interprétation, la décision est présentée comme une contrainte, acceptée uniquement par les mères qui craignent de voir leurs enfants mourir de faim ou celles qui ont peur des conséquences. C'est dans ce contexte désespéré où chacun voit des traîtres partout qu'Eleni, l'Amerikana, parce qu'elle a réussi à faire passer 4 de ses enfants en zone gouvernementale, est arrêtée, torturée, jugée comme traitre par un tribunal populaire et exécutée quelques jours avant la défaite finale de l'AD en Mourgana. La dernière fille, qui n'avait pu s'échapper, car elle était réquisitionnée au travail des champs, sera évacuée comme tous les survivants vers l'Albanie, et rejoindra le reste de la famille aux États-Unis en 1950.

34 Je n'ai pas mentionné ici la caricature des forces de l'ELAS que présente aussi ce livre, car ce n'était pas mon propos. Pour lui tous les communistes sont des simples d'esprit endoctrinés ou des envieux, mais pour apprécier ce texte, il ne faut pas oublier le 
traumatisme de son auteur, un jeune garçon qui voit périr la mère qu'il adorait, le cadre très précis de Lia, village placé malgré lui au cœur d'une situation confuse qui le dépasse, et le fait qu'il présente un cas particulier. Il n'est pas question d'en faire un modèle explicatif à généraliser, il ne faut pas en faire un argument pour juger du recrutement des andartès, du paidomazoma ou de la justice populaire, c'est « un » cas tragique dans une réalité composée de milliers de cas personnels différents qui rappelle simplement que rien n'est tout blanc ou noir.

\section{NOTES}

1. Voir deux études récentes dans le cadre du Programme d'initiative communautaire INTERREG GRECE-ITALIE 2000-2006, "L'image de la Mère entre tradition et époque contemporaine », dans programme 11-003 « Environnement et héritage culturel », mesure 002 « Promotion, rénovation et valorisation de l'héritage historique et culturel d'intérêt commun", réalisées par le TEI d'Épire. Eleni Balaska, Adriani Oikonomou, Chrysostomos Stylios, La femme d'Épire et sa place dans la société de l'Antiquité à l'époque moderne, 33 p. Adriani Oikonomou, Chrysostomos Spilios, La femme d'Épire au travail dans et à l'extérieur de la maison, $30 \mathrm{p}$.

2. Sketchs où de jeunes gens tiennent les rôles féminins, une jeune fille respectable ne saurait monter sur une scène.

\section{RÉSUMÉS}

Ce livre fort contesté présente la vie d'une paysanne d'un village isolé d'Épire depuis son mariage jusqu'à son exécution par l'Armée démocratique en 1948. Vivant dans un milieu ultratraditionnel, mais dans un lieu qui se trouve placé au cœur des conflits, comme toutes ses voisines, elle est plongée dans une série d'événements, incompréhensibles pour elle, qu'elle doit affronter. Quels que soient les partis pris de son auteur, le livre reste un témoignage terrible des souffrances de cette décennie.

This largely contested book presents the life of a woman leaving in an isolated village of Epirus from her marriage till her execution by the Democratic Army in 1948. As she was leaving in an ultra-traditional place and family, in a village located in the center of the conflicts, as all her neighbors, she finds herself in the very heart of many events that she must face even if she can't understand them. Whatever the bias of the author, the book remains a terrible testimony of the sufferings of this decade.

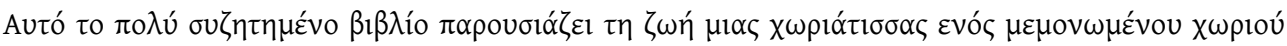

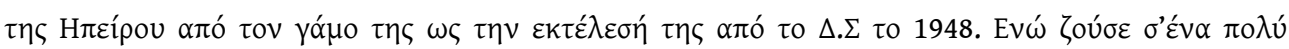

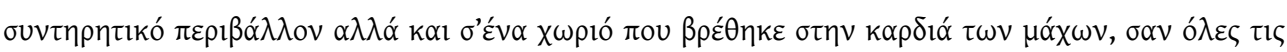




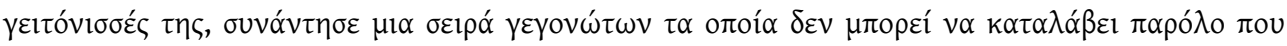

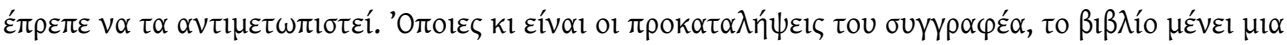

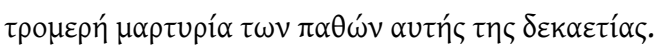

\section{INDEX}

Keywords : women history, paidomazoma, Epirus, second World War, Greek civil war, History Mots-clés : histoire des femmes, Gage Nicholas (1939-), paidomazoma, Tsipouro, ELAS, EDES, UNRRA

motsclesmk ИСТОРИЈАТА НА ЖЕНИТЕ, ПЕДОМАЖОМА, ВТОРАТА СВЕТСКА ВОЈНА, ИСТОРИЈА motsclestr Kadın Tarihi, Pedomazoma, Epir, Ikinci Dünya Savaşı, Tarih

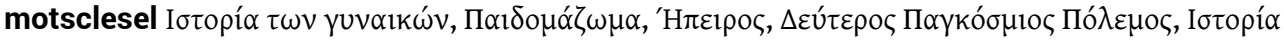
Index géographique : Épire

Thèmes : Histoire, Histoire des femmes

Index chronologique : guerre civile grecque (1946-1949), guerre mondiale (1939-1945) 